\title{
Evaluating the effectiveness of abbreviated breast MRI (abMRI) interpretation- training for mammogram-readers: a multicentre study assessing diagnostic performance, using an enriched dataset
}

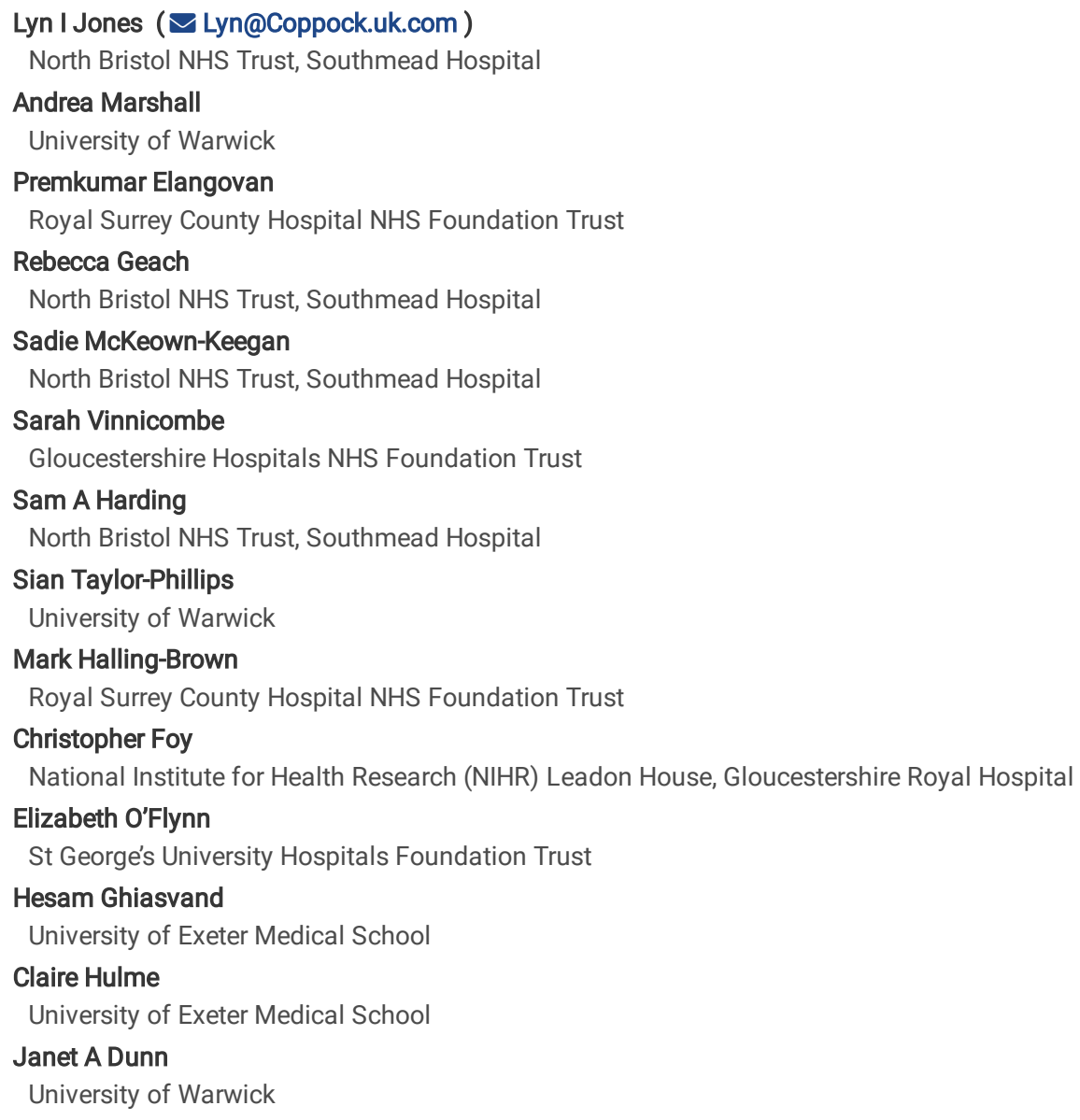

\section{Research Article}

Keywords: Breast cancer, Abbreviated breast MRI, FAST MRI, Education, Training, Diagnostic accuracy

Posted Date: February 21st, 2022

DOl: https://doi.org/10.21203/rs.3.rs-1357525/v1

License: (9) (1) This work is licensed under a Creative Commons Attribution 4.0 International License. Read Full License 


\section{Abstract}

Background: Abbreviated breast MRI (abMRI) is being introduced in breast screening trials and clinical practice, particularly for women with dense breasts. Upscaling abMRI provision requires the workforce of mammogram-readers to learn to effectively interpret abMRI.

The purpose of this study was to examine the diagnostic accuracy of mammogram-readers to interpret abMRI after a single day of standardised small-group training and to compare diagnostic performance of mammogram-readers experienced in full-protocol breast MRI (fpMRI) interpretation (Group 1) with that of those without fpMRI interpretation experience (Group 2).

Methods: Mammogram-readers were recruited from six NHS Breast Screening Programme sites. Small-group hands-on workstation training was provided, with subsequent prospective, independent, blinded interpretation of an enriched dataset with known outcome. A simplified form of abMRI (first-post-contrastsubtracted-images (FAST MRI), displayed as maximum-intensity-projection (MIP) and subtracted slice-stack), was used. Per-breast and per-lesion diagnostic accuracy analysis was undertaken, with comparison across groups, and double-reading simulation of a consecutive screening subset.

Results: 37 readers (Group 1: 17, Group 2: 20) completed the reading task of 125 scans (250 breasts) (total $=9250$ reads). Overall sensitivity was $86 \%$ ( $95 \%$ Confidence Interval (Cl) 84-87\%; 1776/2072) and specificity, 86\% (95\% Cl 85-86\%; 6140/7178). Group 1 showed significantly higher sensitivity (843/952; 89\%; $95 \% \mathrm{Cl} 86-91 \%)$ and higher specificity $(2957 / 3298 ; 90 \%$; 95\%Cl 89-91\%) than Group 2 (sensitivity = 83\%; 95\% Cl 81-85\% (933/1120) p<0.0001; specificity = 82\%; $95 \% \mathrm{Cl} 81-83 \%(3183 / 3880) \mathrm{p}<0.0001)$. Inter-reader agreement was higher for Group 1 (kappa $=0.73 ; 95 \% \mathrm{Cl} 0.68-0.79)$ than for Group 2 (kappa $=0.51 ; 95 \% \mathrm{Cl}$ $0.45-0.56)$. Specificity improved for Group 2, from the first 55 cases $(81 \%)$ to the remaining $70(83 \%)(p=0.02)$ but not for Group 1 ( $90 \%$ to $89 \% \mathrm{p}=0.44$ ); whereas sensitivity remained consistent for both Group 1 (88\% to $89 \%$ ) and Group 2 (83\% to $84 \%)$.

Conclusions: Single day abMRI interpretation-training for mammogram-readers achieved an overall diagnostic performance within benchmarks published for fpMRI but was insufficient for diagnostic accuracy of mammogram-readers new to breast MRI to match that of experienced fpMRI readers. Novice MRI reader performance improved during the reading task, suggesting additional training could further narrow this performance gap.

\section{Background}

Breast cancer screening in most western countries predominantly uses digital mammography technology, with full-protocol breast MRI (fpMRI) used for some high-risk groups. Randomised controlled screening trials of abbreviated breast MRI (abMRI) from the USA(1) and of fpMRI from Europe(2, 3), have demonstrated the potential use of abMRI for screening a wider group of women than those currently screened with fpMRI. In response, abMRI has been introduced into clinical practice to screen women with either mammographically dense breasts or other reasons for being above population-risk of breast cancer(4-10).

The diagnostic accuracy of abMRI is similar to that of fpMRI when reported by professionals expert in fpMRI interpretation(11-19), while shorter acquisition (3-13 minutes abMRI vs. 15-32 fpMRI) and reading times for abMRI (0.6-3 minutes vs. 3-7) promise potential cost savings in comparison with fpMRI(11$14,16,18,20,21)$. However, important unknowns include the feasibility of upscaling abMRI capacity $(20,22)$. Assessing the capacity of the workforce to interpret the additional abMRI scans is an essential part of feasibility assessment.

Learning fpMRI interpretation is an apprenticeship-style process, taking several years to obtain accredited skills. The American College of Radiologists (ACR), European Society of Breast Imaging (EUSOBI) and the UK's National Breast Imaging Academy consider 100 documented/logged fpMRI interpretations over 12 years' accredited learning to be sufficient to demonstrate proficiency in fpMRI reporting(23-25). Internationally, the professional group best placed to augment numbers of existing fpMRI interpreters to read screening abMRI may be readers of screening mammograms, for whom additional training, accreditation and quality assurance would be required $(10,26)$.

A previous single-centre study, of 8 readers from the UK National Health Service Breast Screening Programme (NHSBSP), suggested that NHSBSP mammogram-readers could be effectively trained to interpret abMRI with a single day's one-to-one training(21, 27). Other international publications describe specific abMRI interpretation training for radiologists reading abMRI $(1,28,29)$, but there is little published evidence evaluating abMRI interpretation-training To address this knowledge gap with formal evaluation of abMRI small-group training, our previous study's one-to-one training(21) was adapted to create an electronic training package, delivered as single day, small-group, in person, hands on workstation training. We present the results of a multi-centre study designed to evaluate the impact of the training on mammogram-readers and to compare diagnostic performance of mammogram-readers experienced in fpMRI interpretation with that of those without fpMRI interpretation experience.

\section{Methods}

This study was reviewed and approved by the London-Bromley Research Ethics Committee and by the Health Research Authority (England and Wales)(REC: 19/LO/1473 IRAS:258203), and prospectively registered (ISRCTN:16624917), and all participants gave written informed consent.

Aims

To examine the diagnostic accuracy of mammogram-readers to interpret abMRI after a single day of standardised small-group training and to compare diagnostic performance of mammogram-readers experienced in fpMRI interpretation with that of those without fpMRI interpretation experience.

Study design

Prospective, blinded interpretation of an enriched dataset by multiple readers. 
Participants and setting

NHSBSP multi-professional mammogram-readers, fully qualified to interpret mammograms(30), at 6 sites (NHSBSP screening units) within the South-West Region of England were invited (September-December 2019) and classified as Group 1 if they also interpreted fpMRI in their normal clinical practice, and Group 2 if not. Participants attended a single day of standardised training (October 2019 - January 2020) and then interpreted a test set of abMRI scans (January-July 2020).

Test set

The test set comprised 125 abMRIs with known outcome acquired as fpMRI during 2015: 72 consecutive high-risk screening scans(31) (including two with unilateral cancer) enriched with 53 additional cancer cases from consecutive fpMRI scans acquired at cancer diagnosis (reported as unifocal invasive cancer $\leq 25 \mathrm{~mm}$ or ductal carcinoma in-situ (DCIS) of any size). Of the two cancers within the high-risk screening series of 72 scans, one was detected from the $2015 \mathrm{fpMRI}$ (screen-detected) but the other was not recognised in 2015 (interval cancer). All cancers had histological confirmation and non-cancer scans had 2-year minimum-follow-up. Test set composition, imaging and display protocol were previously described(21) (abMRI specification reproduced in Appendix 1). Of 125 abMRIs in the dataset, 54 had biopsy-confirmed unilateral cancer and one bilateral (56 breasts with cancer) and 2 women had two separate tumours identified in the same breast, giving a total of 58 cancers reported in the ground truth(21).

Electronic format

Software was developed to display abMRI (RiViewer)(32) using a simplified display protocol (first-post-contrast-subtracted-images (FAST MRI) displayed as maximum-intensity-projection (MIP) and stacked, subtracted slices). Biopsy-proven cancers were drawn onto images electronically as ground truth. During hands-on workstation training (29 training abMRI scans), learners could discover the ground truth at the touch of a button, giving instant feedback (formative assessment).

The software contained an automatic timer to measure interpretation times.

The same software displayed the test set of 125 abMRIs(21). The test set and the set of training scans were mutually exclusive. Training and test set MRIs were from a single centre but acquired during different years, from different women. The test set was presented to each reader in a different random order, and readers were unable to access the ground truth of the test set at any time (summative assessment).

Standardised training

The structured training package $(21,27)$ was adapted to enable in person, small-group training, delivered by two radiologists experienced in fpMRI reporting (Appendix 2: example study day agenda). Small group presentations on aspects of abMRI interpretation alternated with guided hands-on workstation sessions to enable learners to practice image manipulation and abMRI interpretation on the training set of 29 abMRI scans. The presentations included multiple additional illustrative examples of abMRI images depicting specific learning points. These examples were taken from MRI scans not included in either the training or test sets.

Throughout the training, mammogram-readers' prior knowledge was utilised and activated by repeated reference to similarities and differences between the two breast imaging modalities (abMRI and mammogram) and the varied appearances of cancer, and of other common breast pathologies, as displayed by each modality (33).

The training set was presented in batches, of increasing difficulty, as guided hands-on workstation practice during which readers could discover the ground truth at the touch of a button, giving instant feedback to aid their learning (formative assessment).

Readers were taught how to classify abMRI scans according to the UK 5-point breast imaging classification specified for screening fpMRI in women at higher risk of breast cancer within $\operatorname{NHSBSP}(34,35)$.

Test set interpretation

Subsequent to completion of their training, readers interpreted the test set of 125 abMRIs(21), blinded to all other information (clinical history, previous imaging, histology and other readers' interpretations). Readers were told to expect more cancers than in usual screening practice but no other indication of the number of cancers was given. The test set was presented to each reader in a different random order and readers were unable to access the ground truth at any time.

Sample-size calculation

Using the results of a previous single-centre study(21), a dataset of 250 breasts (125 women) allowed the lower $95 \%$ confidence limit of the inter-rater reliability to be estimated to within 0.07 with a minimum of 6 readers/group and a proportion of cancers of 0.22 . Thus, we aimed for a minimum of 12 readers: 6 in each group.

\section{Statistical analysis}

Per-breast analysis of the frequency of results against true outcome was obtained overall and for each reader. Sensitivity and specificity of readers' abMRI classification with the true outcome were determined and differences across reader groups assessed using a multilevel-generalised-mixed model to account for multiple readers per scan and the dependence between breasts. The inter-reader variability and the agreement between readers and the true outcome were 
assessed using Cohen's $\mathrm{k}$ coefficient to account for the probability of agreement occurring by chance. Classifications 4 and 5 were considered indicative of cancer, and classifications 1-3 considered a normal result.

Interpretation times were compared across reader groups (Wilcoxon rank-sum). If readers returned to a scan on multiple occasions, interpretation times were calculated as total-time spent on the scan.

To assess whether readers' performance improved during the assessment task, the initial 55 scans interpreted by each reader (first set) and the subsequent 70 (second set) were compared overall and for each group.

A per-lesion analysis was also undertaken. Lesion localisation fraction (LLF) was calculated (number of true positives divided by total number of true cancers). For each reader group, a weighted jackknife alternative free-response receiver operator characteristics (JAFROC) curve was determined using the abMRI classifications for identified cancers, plotting the LLF against the false positive fraction (fraction of normal breast with at least one false positive on its image). The empirical areas under the equally weighted JAFROC curve were used as figures of merit (FOM). Reader averaged FOM for each group were compared using an analysis of variance (ANOVA) test. Data were analysed using SAS statistical software and "RJafroc" package within the R software.

Lastly, as a per-breast analysis, to simulate double reading (standard UK screening practice), results were calculated from randomly selecting two readers, and a third for arbitration of disagreement(36).

\section{Results}

Thirty-seven participants (17 mammogram-readers experienced in fpMRI interpretation (Group 1) and 20 mammogram-readers without previous experience of fpMRI interpretation (Group 2)) completed both the training and the subsequent reading task, of the 125 abMRI dataset (250 breasts), giving a total of 9250 reads. Figure 1 is the flow chart of reader recruitment, and Table 1 details the professional roles and experience of the readers in each of the two groups. The training days were delivered by two authors ( $L J$ and RG). Participant readers each attended a single training day and were trained in groups of 1-7 (median $4)$.

Table 1

Demographics of participant mammogram-readers

\begin{tabular}{|c|c|c|}
\hline & Group 1 & Group 2 \\
\hline \multicolumn{3}{|l|}{ Professional Title } \\
\hline Advanced Practitioner & 0 & $12^{*}$ \\
\hline Consultant Radiographer & 0 & 5 \\
\hline Breast Clinician & $2^{*}$ & 4 \\
\hline Consultant Radiologist & $17 *$ & 1 \\
\hline \multicolumn{3}{|l|}{ Professional Experience } \\
\hline Number of years interpreting mammograms: median (range) & $10(1-25)$ & $6(<1-19)$ \\
\hline Number of mammograms interpreted each year: median (range) & $6,000(3,000-13,000)$ & $5,000(4,500-11,600)$ \\
\hline Participant readers who interpret digital breast tomosynthesis in normal clinical practice & 13 & 10 \\
\hline Number of years interpreting breast MRI: median (range) & $6(0.5-20)$ & $\mathrm{N} / \mathrm{A}$ \\
\hline Number of full protocol breast MRI scans interpreted each year: median (range) & $100(40-350)$ & $\mathrm{N} / \mathrm{A}$ \\
\hline Total numbers of participant readers who attended the FAST MRI study day & 19 & 22 \\
\hline
\end{tabular}

*In total 4 participant readers attended the training session but did not complete the follow-up dataset, namely, one Consultant Radiologist, one Breast Clinician and two Advanced Practitioners.

Per-breast analysis

The per-breast analysis comparing readers' MRI classification with the true outcome (cancer or normal) showed an overall sensitivity of $86 \%$ (95\% Cl $84-87 \%$; $1776 / 2072)$ and specificity of $86 \%$ (95\% Cl $85-86 \% ; 6140 / 7178)$.

Results for readers in Group 1 showed significantly higher sensitivity (843/952; 89\%; 95\% Cl 86-91\%) and higher specificity (2957/3298; 90\%; 95\%Cl 89-91\%) than Group 2 (sensitivity $=83 \% ; 95 \% \mathrm{Cl} 81-85 \%(933 / 1120) \mathrm{p}<0.0001$; specificity $=82 \% ; 95 \% \mathrm{Cl} 81-83 \%(3183 / 3880) p<0.0001)$. Inter-reader agreement was also higher for Group 1 (kappa $=0.73 ; 95 \% \mathrm{Cl} 0.68-0.79)$ than for Group $2(\mathrm{kappa}=0.51 ; 95 \% \mathrm{Cl} 0.45-0.56)(\mathrm{Table} 2)$. 
Table 2

Comparison of readers' MRI classification against true outcome (per breast)

\begin{tabular}{|c|c|c|c|c|c|}
\hline & & \multicolumn{4}{|c|}{ Ground Truth Result } \\
\hline & & Cancer & Normal & Total & Kappa (95\% Cl) \\
\hline & \multicolumn{5}{|l|}{ Reader Classification } \\
\hline \multirow[t]{3}{*}{ All Readers } & Cancer & 1776 & 1038 & 2814 & \\
\hline & No cancer & 296 & 6140 & 6436 & \\
\hline & Total & 2072 & 7178 & 9250 & $0.63(0.61-0.65)$ \\
\hline \multirow[t]{3}{*}{ Group 1} & Cancer & 843 & 341 & 1184 & \\
\hline & No cancer & 109 & 2957 & 3066 & \\
\hline & Total & 952 & 3298 & 4250 & $0.72(0.70-0.74)$ \\
\hline \multirow[t]{3}{*}{ Group 2} & Cancer & 933 & 697 & 1630 & \\
\hline & No cancer & 187 & 3183 & 3370 & \\
\hline & Total & 1120 & 3880 & 5000 & $0.56(0.54-0.59)$ \\
\hline
\end{tabular}

The Receiver Operating Characteristics Curve of the per-breast individual reader performance demonstrates some overlap between the two groups (Fig. 2).

There was a significant improvement in the specificity of the Group 2 readers from the first 55 scans interpreted to the remaining 70 scans from $81-83 \%$ ( $p=$ $0.02)$, whereas their sensitivity remained fairly consistent from $83-84 \%(p=0.59)$. There were no significant improvements for the expert readers of Group 1 , neither in sensitivity (from $88-89 \%(p=0.54)$ ) nor in specificity (from $90-89 \%(p=0.44)$ ) (Fig. 3 ).

Time taken to report

The median time taken for individual readers to interpret each abMRI scan was 29 seconds less for Group 1 (median 86 seconds, range $17-1145$ seconds, interquartile range 60-127) than for Group $2(115,17-10003,76-173 p<0.0001)$ (Fig. 4). There were 25 (of 37 ) readers that returned to a total of 75 (of 125 ) scans on multiple times (range 1 to 15 scans) and there were 7 records (out of a total of 9250) where a reader took more than 1000 seconds to interpret. The interpretation time for both Group 1 and Group 2 readers decreased from the first 55 scans interpreted to the subsequent 70 (Group 1 median interpretation time decreased by 19.86 seconds $(p<0.0001)$, Group 2 by $31.11(p<0.0001))($ Table 3$)$.

Table 3

Interpretation times compared across the sets of FAST MRI scans, overall and for each group of readers

\begin{tabular}{|c|c|c|c|}
\hline Total time & Overall & Group 1 & Group 2 \\
\hline \multicolumn{4}{|l|}{ First Set } \\
\hline Number & 2035 & 935 & 1100 \\
\hline \multicolumn{4}{|l|}{ Total time } \\
\hline Median & 115.33 & 98.04 & 133.40 \\
\hline Interquartile range & $74.25-173.49$ & $65.56-142.26$ & 84.89-196.05 \\
\hline Range & $17.14-10003.38$ & $17.14-1144.79$ & $22.72-10003.38$ \\
\hline \multicolumn{4}{|l|}{ Second Set } \\
\hline Number & 2590 & 1190 & 1400 \\
\hline \multicolumn{4}{|l|}{ Total time } \\
\hline Median & 89.70 & 78.18 & 102.29 \\
\hline Interquartile range & $62.26-136.11$ & $55.23-114.16$ & $71.62-150.54$ \\
\hline \multirow[t]{2}{*}{ Range } & $16.95-1645.50$ & $117.99-796.06$ & $16.95-1645.50$ \\
\hline & $P<0.0001$ & $P<0.0001$ & $P<0.0001$ \\
\hline
\end{tabular}

Per-lesion analysis

There were 58 biopsy-confirmed cancer lesions in the dataset, equating to a total of 2146 decisions made by the 37 readers. The LLF was $83 \%$ (1783/2146) overall, 86\% (847/986) for the Group 1 readers and 81\% (936/1160) for the Group 2 readers. The reader averaged weighted JAFROC FOM was 0.93 (95\% Cl 0.92-0.94) overall. The FOM for Group 1 readers of 0.95 ( $95 \% \mathrm{Cl} 0.95-0.96)$ was significantly higher than for Group $2(0.91 ; 95 \% \mathrm{Cl} 0.89-0.93)$; $\mathrm{p}=0.004)$ (Fig. 5). 
Per-woman analysis

On a per-woman basis, the 125 women (whose abMRI scans comprised the test set) were reported by the 37 readers, giving a total of 4625 reads. Thirty eight percent of the women (1744/4625) were correctly identified as having cancer and therefore would have been correctly recalled if their abMRIs had been single read, while $41 \%$ women (1898/4625) were correctly identified as not having cancer and would not have been recalled. A further $10 / 4625$ ( $<1 \%)$ women with cancer would have been recalled, but incorrectly, based on a different lesion. In total, 15\% women (692/4625) would have been incorrectly recalled but found not to have cancer and $6 \%$ women $(281 / 4625)$ with cancer would have been missed and not recalled if single read (Fig. 6). These figures represent single reading of the test set by all readers (Group 1 and Group 2) and equate to a per-woman sensitivity of $86 \%$ (1744/2035) and specificity of $73 \%$ (1898/2590), with $14 \%$ false negatives $(281 / 2035)$ and $27 \%$ false positives $(692 / 2590)$.

Double reading simulation analysis for the consecutive series of screening cases

The enriched test set of 125 abMRI scans included 72 consecutive screening cases and the results for these 72 women alone (consecutive screening subset) were re-analysed on a per-breast basis to simulate double reading (standard practice in NHSBSP). Using two random readers and a third for arbitration when there was disagreement, there were 144 breast results comprising two breasts with biopsy-proven cancer and 142 without cancer (at least 2-year normal follow up).

There were 124/144 (86\%) breasts correctly identified as not having cancer, and both breasts with cancer were correctly identified as having cancer ( $2 / 144$ (1\%)). There were no false negatives. However, 18/144 (13\%) breasts were incorrectly identified as having cancer. Hence sensitivity was $100 \%$ (85\% Cl 16$100 \%$ ) with $87 \%(95 \% \mathrm{Cl} 81-92 \%)$ specificity.

Of the 18 false positive breasts, the original fpMRI reports (unavailable to readers) contained the following information (unavailable to the blinded readers in this study): 6 lesions were noted on the original $2015 \mathrm{fpMRI}$ report to be unchanged since a previous fpMRI, 3 had previously been biopsied and were therefore known to be benign at the time of reporting in 2015, 1 had a recent biopsy noted in the 2015 report that explained the positive finding and 2 had been recalled in 2015 from the fpMRI for the same finding which was subsequently demonstrated as benign by either biopsy or follow up.

\section{Discussion}

Summary of findings

Following a single day of standardised, small-group training, mammogram-readers' overall diagnostic performance at abMRI interpretation achieved benchmarks set for fpMRI by the American College of Radiology's Breast Imaging Reporting and Data System (BI-RADS) for both sensitivity (86\% achieved vs. $>80 \%$ BI-RADS benchmark(37)), and specificity ( $86 \%$ achieved vs. $>85 \%$ BI-RADS benchmark(37)).

The performance of readers experienced in interpreting fpMRI (Group 1) was significantly better than that of mammogram-readers without previous experience in fpMRI interpretation (Group 2): sensitivity ( $<0.0001)$, specificity $(p<0.0001)$, and inter-reader variability (non-overlapping $95 \%$ Cls).

The performance of the novice readers of Group 2 improved during the reading task $(p=0.02)$ whereas that of the expert readers of Group 1 did not. This improvement in performance of Group 2 readers occurred despite them receiving no feedback about the ground truth of scans during the reading task.

Literature comparison with reader results in this study - diagnostic accuracy

A European multi-reader study of Ultrafast breast MRI interpretation of an enriched dataset by 7 breast radiologists, with 6-15 years' experience in fpMRI interpretation, compared diagnostic performances at Ultrafast breast MRI and fpMRI. Their readers' diagnostic performance at Ultrafast (sensitivity: $84 \%$ and specificity $82 \%$ ) was similar to that of our novice Group 2 readers, who had no previous experience in breast MRI interpretation (sensitivity: $83 \%$ and specificity: $82 \%)$. Their inter-reader agreement (kappa $=0.73)$ was similar to that of our fpMRI-experienced, Group 1 readers $(0.71)$ while our FOM from the perlesion JAFROC analysis (Group 1: 0.95, Group 2: 0.91 and overall: 0.93$)$ compared well with their non-localised AUC (0.89)(38).

Single reading diagnostic performance at abMRI of the novice Group 2 readers in the current study was similar to published figures for diagnostic performance at fpMRI for radiologists, experienced in breast MRI interpretation, in community screening practice in the USA (13,000 fpMRI examinations reported by the Breast Cancer Surveillance Consortium (BCSC): sensitivity: $83 \%$ Group 2 vs. $81 \%$ BCSC and specificity: $82 \%$ Group 2 vs. $83 \%$ BCSC(39).

The published EA1141 trial reported diagnostic accuracy for abMRI, single read (standard USA practice) by experienced fpMRI readers who had successfully completed the Society of Breast MRI's abMRI interpretation course. The diagnostic accuracy its abMRI readers achieved (sensitivity: $95.7 \%$ and specificity: $86.7 \%)(1)$ is similar to the results of our double reading simulation analysis of the consecutive screening subset of scans within our test set (Groups $1 \& 2$ readers combined) of $100 \%$ sensitivity and $87.3 \%$ specificity. Double reading is standard UK practice.

In a study of 116 Australian breast radiologists, interpreting screening mammograms for population-risk women under test conditions, outside clinical practice(40), their overall JAFROC score was 0.78 (95\% $\mathrm{Cl} 0.77-0.80)$ while that of the subset of radiologists who read $>5000$ mammograms/year was higher: 0.86 (95\% $\mathrm{Cl} 0.83-0.88)$. Both these figures are lower than the equivalent figures (FOM) obtained for FAST MRI by our readers (Group 1: 0.95 , Group 2: 0.91 and overall: 0.93 ). In addition, our readers' figures for LLF (overall: 0.83 ; Group 1: 0.86 and Group 2: 0.81 ) are also considerably higher than the equivalent figures for location sensitivity achieved for mammography by the Australian radiologists (overall: 0.56 , and for the subset of radiologists reading $>5000$ mammograms/year: 0.59). These differences in LLF highlight the greater inherent sensitivity in the technique of FAST MRI in comparison with mammography, as FAST MRI was designed to expand the indications for breast MRI into populations of women currently screened with mammography, such as women with mammographically dense breasts who are otherwise at population risk of breast cancer but whose cancers are often missed by mammography( 1,3$)$. 
Literature comparison with reader results in this study - reading times

The median reading times, of both the expert-readers (Group 1) and novice-readers (Group 2) in the current study, fall within the range of times to interpret abMRI reported in the literature $(11,13,14,16,18)$, despite the automated recorded timings including time taken by readers to electronically complete required answers for each case about background parenchymal enhancement and motion artefact. Group 2, on average, took approximately a third longer than Group 1 to interpret abMRI (median 115 seconds vs. 86). Whilst time taken to interpret abMRI significantly shortened during the reading task for both Groups 1 and 2 , allowance for longer interpretation times by Group 2 readers should be factored into workforce planning around this new technology.

Study limitations

Limitations of this study include that an enriched dataset was used, as discussed in a previous publication of this dataset(21), and that the dataset was not read within clinical practice, exposing the readers to a negative "laboratory effect" on their performance(41).

In the current study we included an additional analysis of the small, consecutive screening series(31) (72 abMRI scans, a subset of the enriched test set), without enrichment. Simulated double-reading, per-breast analysis of this consecutive screening series subset of scans was performed using the relatively large number of independent blinded reader interpretations obtained. However, although we used the proxy for arbitration that was available to us, we understand that arbitration in practice would be different and could therefore yield different results.

Double reading simulation

The double reading simulation enabled a tentative prediction of the potential diagnostic accuracy that using abMRI, double-read by trained mammogramreaders (those with previous experience of fpMRI interpretation and those without) might achieve if used to screen this population.

Double reading simulation analysis of the consecutive screening subset suggested correct identification of both of the 2 breasts with cancer for a recall rate of $20 / 144(14 \%)$. In our study, double reading of abMRI was entirely blinded to past history and previous imaging, unlike in clinical practice. Of the $18 / 144$ false positive assessments made by double-reading simulation in this study, interrogation of the original $2015 \mathrm{fpMRI}$ reports, revealed past history information and/or previous imaging information (unavailable to our readers during the study) that for 10/18 breasts had enabled avoidance of recall from fpMRI in 2015. Thus, if past history and previous imaging had been available to the readers of this study at the time of abMRI interpretation, they may also have been able to avoid recall for these scans and the recall rate for double-reading simulation may have been 8/144 (6\%), whilst still correctly identifying both the true cancers in this consecutive screening subset $(2 / 2)$.

There is evidence that in the practice of screening mammography, reader diagnostic performance is related to the annual number of mammograms read and the number of years of experience in mammogram interpretation(40). It may be that the diagnostic performance achieved by our novice Group 2 readers from a single day of training could be sufficient for them to join their fpMRI-experienced Group 1 colleagues in contributing to double reading of abMRI, provided there were initial individual credentialing by sensitivity and specificity threshold and ongoing audit and performance assessment, similar to that currently in place for mammogram-readers within NHSBSP (Breast Screening Information System (BSIS)(42) and Personal Performance in Mammographic Screening (PERFORMS)(43)). These systems could chart the improvement likely to occur in mammogram-readers' performance with increasing abMRI-interpretation experience.

Implications of the research

Figure 7 illustrates an example cancer case from the test set. The $25 \mathrm{~mm}$ Grade 2 carcinoma of no special type was occult mammographically and is not well demonstrated on the FAST MRI MIP image but clearly visible on images from the FAST MRI stack of slices. It was correctly identified as a cancer by $17 / 17$ Group 1 readers and 17/20 Group 2 readers during the study. The enriched test set used in this study was developed to be a challenging test of performance for novice abMRI readers(21) and included 56 breasts with cancer, of which 25/56 had lobular histology, more difficult to detect at mammography than cancers with other histology(44) and an additional 18/56 breasts with cancers that were mammographically occult in clinical practice (including the case illustrated in Fig. 7).

The improvement in performance demonstrated during the reading task by our novice readers indicates the presence of a learning curve for this group and the results of this study suggest it is likely that additional training will enhance the performance of these readers in terms of both diagnostic accuracy and interpretation speed. Further research is needed, to explore additional training of mammogram-readers new to breast MRI interpretation, to map their learning curve.

The results of this study showed, as expected, that a single day of abMRI interpretation-training is insufficient for the diagnostic accuracy of mammogramreaders new to breast MRI interpretation (Group 2) to match that of those experienced in fpMRI interpretation (Group 1). However, overall diagnostic accuracy for single reading by the two groups of readers combined was within published benchmarks, and the single day of training enabled the multi-professional mammogram-readers new to breast MRI interpretation (Group 2) to achieve diagnostic performance comparable with that published for radiologists experienced in breast MRI in community screening practice(39).

The similarity of the results achieved by the double reading simulation of the consecutive screening series subset of the test set to those achieved in the published EA1141 trial of abMRI (in which scans were single reported by radiologists experienced in fpMRI following additional abMRI interpretation-training) suggests that the current study's standardised single-day training may be sufficient for mammogram-readers to commence their contribution to double reading of abMRI with appropriate initial credentialing and ongoing audit of performance. 
Given that breast MRI is much better at detecting high grade, aggressive cancers at a smaller size than mammography $(2,3,45)$ and early detection of breast cancer improves survival $(46,47)$, upscaling abMRI provision and augmentation of the current fpMRI interpretation workforce through the development of standardised, effective training and performance-evaluation is a priority for the specialty. Prospective feasibility research to investigate current uncertainties around recall rates and rates of image-guided core biopsy, vacuum-assisted biopsy and MRI-guided biopsy will be another necessary research step to enable cost-effectiveness analysis of the use of abMRI as a screening tool and will inform decisions by policy makers about the potential introduction of abMRI into future clinical screening practice.

\section{Conclusions}

Single day abMRI interpretation-training achieved diagnostic performance, at single read, for NHSBSP mammogram-readers within benchmarks published for fpMRI.

The single day of training was insufficient for diagnostic accuracy of mammogram-readers new to breast MRI to match that of experienced fpMRI readers but may be sufficient for their contribution to double-reading.

Performance of novice abMRI readers showed in-task improvement, indicating a learning curve (potential for improvement with additional training).

\section{Abbreviations}

abMRI = Abbreviated breast Magnetic Resonance Imaging

ACR = American College of Radiology

ANOVA = analysis of variance

AUC $=$ Area under a curve

BCSC $=$ Breast Cancer Screening Consortium

BSIS = Breast Screening Information Service

BI-RADS = American College of Radiology's Breast Imaging Reporting and Data System

$\mathbf{C l}=$ Confidence interval

DCIS = Ductal carcinoma in-situ

EUSOBI = European Society of Breast Imaging

FAST MRI = First post contrast subtracted images (breast MRI)

FOM = Figures of merit

fpMRI = Full protocol breast Magnetic Resonance Imaging

IRAS = Integrated Research Application System for applications to the Health Research Authority and the Research Ethics Committee

ISRCTN = International Standard Randomised Controlled Trial Number (however, over the years the scope of the registry has widened beyond randomised controlled trials to include any study designed to assess the efficacy of health interventions in a human population.)

JAFROC $=$ jack-knife alternative free-response receiver operator characteristics

LLF $=$ Lesion localisation fraction

MIP = Maximum intensity projection image

MRI = Magnetic Resonance Imaging

NHSBSP $=$ National Health Service (United Kingdom) Breast Screening Programme

PERFORMS $=$ Personal Performance in Mammographic Screening

REC $=$ Research Ethics Committee

UK = United Kingdom of Great Britain and Northern Ireland

USA $=$ United States of America

\section{Declarations}


Ethics approval and consent to participate: In accordance with the Declaration of Helsinki on research involving human participants, this study was reviewed and approved by the London-Bromley Research Ethics Committee (REC: 19/LO/1473) and by the Health Research Authority and Health and Care Research Wales (IRAS:258203). The study was prospectively registered (ISRCTN:16624917).

All participants gave informed consent (written) to their participation in the study.

Consent for publication: Written consent was given, for publication of the images that constitute Figure 7 of this manuscript, by the individual from whose MRI scan the images were taken.

Availability of data and materials: The dataset generated and analysed during the current study is not yet publicly available because it is currently being developed into a publicly shareable format. Instead, it is available from the corresponding author on reasonable request.

Funding: This manuscript presents independent research funded by the National Institute for Health Research (Research for Patient Benefit (RfPB), Refinement and piloting of a training programme within the NHS Breast Screening Programme (NHSBSP) workforce of image readers to enable standardised interpretation of a shortened magnetic resonance imaging scan (MRI) of the breast called FAST MRI to support the delivery of a future multicentre trial of FAST MRI versus mammogram for breast cancer screening, PB-PG-1217-20008). The views expressed in this publication are those of the authors and not necessarily those of the NHS, the National Institute for Health Research (NIHR) or the Department of Health and Social Care.

Author STP is supported by an NIHR Career Development Fellowship (CDF - 2016-09-018). The views expressed in this manuscript are those of the authors and not necessarily those of the NIHR or the Department of Health and Social Care.

Competing interests: Other than the funding sources declared above, the authors declare that they have no other competing interests.

Authors' contributions: LJ, CF, STP and JD contributed substantially to the conception of the work. LJ, JD, CF, RG, SH, AM, PE, SV, STP, HG and CH had substantial input to the study design. PE, MHB, LJ and RG contributed substantially to the creation of new software used in the study. SMK, PE, AM, LJ, and RG had substantial input to the acquisition of data, while AM and PE conducted the data analysis and LJ, AM, STP, SV, EOF and JD contributed substantially to the data interpretation. LJ and AM drafted the work and subsequently, with additional help from STP, SV, RG, SH, CH and EOF, substantially revised it.

All authors have approved the submitted version of this manuscript and have agreed both to be personally accountable for the author's own contributions and to ensure that questions related to the accuracy or integrity of any part of the work, including ones in which the author was not personally involved, are appropriately investigated, resolved, and the resolution documented in the literature.

The corresponding author is LJ.

\section{Acknowledgements}

This study was performed on behalf of the FAST MRI Study Group which at the time of this study, in addition to the authors, comprised Christiane Kuhl, Jennifer Wookey, Janice Rose, Victoria Taylor, John Gifford, Rosie Gray, Thomas William-Jones, Karen Litton, Simon Lloyd, Jim Steel, Elisabeth Kutt, Alexandra Valencia, Alice Pocklington, Anjum Mahatma, Helen Massey, Gillian Clark, Clare McLachlan, Gemini Beckett, Clare Alison, Miklos Barta, Claudia Betancourt, Julie Bramwell, Nichola Bright, Helen Burt, Louise Cann, Jane Ceney, Eleanor Cornford, Diana Dalgliesh, Sarah Doyle, Sarah Fearn, Dagmar Godden, Zoe Goldthorpe, Lucinda Hobson, Paula Hynam, Emma Jackson, Margaret Jenkin, Beckie Kingsnorth, Katherine Klimczak, Alice Moody, Sarah Perrin, Alison Peters, Elizabeth Preston, Anne Ratsey, Richard Sidebottom, Lesley Stephenson, Michelle Taylor, Erika Toth, Frances Vincent, Sharon Watkin, Sue Widdison, Jennifer Williams, Karen Wilmot, Sravya Singamaneni, Zsolt Friedrich, Joanne Robson, Elizabeth Cullimore and Anna Mankelow.

The authors wish to thank the Breast Unit Support Trust (BUST) and the Independent Cancer Patients' Voice (ICPV) charities and the NIHR Research Design Service (RDS) for their invaluable support.

Publication declaration: This research was initially presented as a poster at the San Antonio Breast Cancer Symposium in December 2020 (abstract published on the SABCS website as PS3-31 https://www.abstractsonline.com/pp8/\#!/9223/session/43). It was subsequently presented as posters P14 and P18 at Symposium Mammographicum in February 2021 and has since been published as two abstracts (P14 and P18), amongst the other Meeting Abstracts for Symposium Mammographicum 2021, in Breast Cancer Research 2021, 23(Suppl 1):67 https://doi.org/10.1186/s13058-021-01443-6.

This research has not otherwise been published previously.

\section{References}

1. Comstock CE, Gatsonis C, Newstead GM, Snyder BS, Gareen IF, Bergin JT, et al. Comparison of Abbreviated Breast MRI vs Digital Breast Tomosynthesis for Breast Cancer Detection Among Women With Dense Breasts Undergoing Screening. JAMA - J Am Med Assoc [Internet]. 2020;323(8):746-56. Available from: https://jamanetwork.com/journals/jama/fullarticle/2761645

2. Kuhl CK, Strobel K, Bieling H, Leutner C, Schild HH, Schrading S. Supplemental Breast MR Imaging Screening of Women with Average Risk of Breast Cancer. Radiology [Internet]. 2017;283(2):361-70. Available from: http://pubs.rsna.org/doi/10.1148/radiol.2016161444

3. Bakker MF, De Lange S V., Pijnappel RM, Mann RM, Peeters PHM, Monninkhof EM, et al. Supplemental MRI screening for women with extremely dense breast tissue. N Engl J Med [Internet]. 2019;381(22):2091-102. Available from: https://www.nejm.org/doi/full/10.1056/NEJMoa1903986 
4. Choi B, Choi N, Kim M, Yang J-H, Yo Y, Jung H. Usefulness of abbreviated breast MRI screening for women with a history of breast cancer surgery. Breast Cancer Res Treat [Internet]. 2018;167(2):495-502. Available from: https://doi.org/10.1007/s10549-017-4530-z

5. Park KW, Han SB, Han B-K, Ko ES, Choi JS. MRI surveillance for women with a personal history of breast cancer: comparison between abbreviated and full diagnostic protocol. Br J Radiol [Internet]. 2020;93:20190733. Available from: https://doi.org/10.1259/bjr.20190733

6. An YY, Kim SH, Kang JB, Young JS, Jeon YW. Feasibility of abbreviated magnetic resonance imaging (AB-MRI) screening in women with a personal history ( PH ) of breast cancer. PLoS One [Internet]. 2020;15(3):1-13. Available from: https://doi.org/10.1371/ journal.pone.0230347

7. Plaza MJ, Perea E, Sanchez-gonzalez MA. Abbreviated Screening Breast MRI in Women at Higher-than-Average Risk for Breast Cancer with Prior Normal Full Protocol MRI. J Breast Imaging [Internet]. 2020;2(4):343-51. Available from: https://doi.org/10.1093/jbi/wbaa032

8. Weinstein SP, Korhonen K, Cirelli C, Schnall MD, Mcdonald ES. Abbreviated Breast Magnetic Resonance Imaging for Supplemental Screening of Women With Dense Breasts and Average Risk original reports abstract. J Clin Oncol. 2020;38(33):3874-82.

9. Partovi S, Sin D, Lu Z, Sieck L, Marshall H, Pham R, et al. Fast MRI breast cancer screening - Ready for prime time. Clin Imaging [Internet]. 2020;60(2):160-8. Available from: https://doi.org/10.1016/j.clinimag.2019.10.013

10. Grimm LJ, Mango VL, Harvey JA, Plecha DM, Conant EF. Implementation of Abbreviated Breast MRI for Screening: AJR Expert Panel Narrative Review. Am J Roentgenol [Internet]. 2021;(published online ahead of print 11th August 2021):1-11. Available from: https://doi.org/10.2214/AJR.21.26349

11. Geach R, Jones LI, Harding SA, Marshall A, Taylor-Phillips S, Mckeown-keegan S, et al. The potential utility of abbreviated breast MRI (FAST MRI) as a tool for breast cancer screening: a systematic review and meta-analysis. Clin Radiol [Internet]. 2021;76:154.e11-154.e22. Available from: https://doi.org/10.1016/j.crad.2020.08.032

12. Baxter GC, Selamoglu A, Mackay JW, Bond S, Gray E, Gilbert FJ. A meta-analysis comparing the diagnostic performance of abbreviated MRI and a full diagnostic protocol in breast cancer. Clin Radiol [Internet]. 2021;76:154.e23-154.e32. Available from: https://doi.org/10.1016/j.crad.2020.08.036

13. Kuhl CK, Schrading S, Strobel K, Schild HH, Hilgers RD, Bieling HB. Abbreviated breast Magnetic Resonance Imaging (MRI): First postcontrast subtracted images and maximum-intensity projection - A novel approach to breast cancer screening with MRI. J Clin Oncol [Internet]. 2014;32:2304-10. Available from: https://ascopubs.org/doi/10.1200/JC0.2013.52.5386

14. Chen SQ, Huang M, Shen YY, Liu CL, Xu CX. Application of Abbreviated Protocol of Magnetic Resonance Imaging for Breast Cancer Screening in Dense Breast Tissue. Acad Radiol [Internet]. 2017;24(3):316-20. Available from: http://dx.doi.org/10.1016/j.acra.2016.10.003

15. Jain M, Jain A, Hyzy MD, Werth G. FAST MRI breast screening revisited. J Med Imaging Radiat Oncol [Internet]. 2017;61(1):24-8. Available from: https://doi.org/10.1111/1754-9485.12502

16. Panigrahi B, Mullen L, Falomo E, Panigrahi B, Harvey S. An Abbreviated Protocol for High- risk Screening Breast Magnetic Resonance Imaging: Acad Radiol [Internet]. 2017;24(9):1132-8. Available from: http://dx.doi.org/10.1016/j.acra.2017.03.014

17. Dialani V, Tseng I, Slanetz P, Fein-Zachary V, Phillips J, Karimova E, et al. Potential role of abbreviated MRI for breast cancer screening in an academic medical center. Breast J [Internet]. 2019;25:604-11. Available from: https://doi.org/10.1111/tbj.13297

18. Harvey SC, Di Carlo PA, Lee B, Obadina E, Sippo D, Mullen L. An Abbreviated Protocol for High-Risk Screening Breast MRI Saves Time and Resources. J Am Coll Radiol [Internet]. 2016;13(11):374-80. Available from: http://dx.doi.org/10.1016/j.jacr.2015.08.015

19. Hernández ML, Osorio S, Florez K, Ospino A, Díaz GM. Abbreviated magnetic resonance imaging in breast cancer: A systematic review of literature. Eur J Radiol Open [Internet]. 2021;8:100307. Available from: https://doi.org/10.1016/j.ejro.2020.100307

20. Vinnicombe S, Harvey H, Healy N. Introduction of an abbreviated breast MRI service in the UK as part of the BRAID trial: practicalities, challenges and future directions. Clin Radiol [Internet]. 2021;76(6):427-33. Available from: https://doi.org/10.1016/j.crad.2021.01.020

21. Jones LI, Geach R, Harding SA, Foy C, Taylor V, Marshall A, et al. Can mammogram readers swiftly and effectively learn to interpret first post-contrast acquisition subtracted (FAST) MRI, a type of abbreviated breast MRI?: a single centre data-interpretation study. Br J Radiol. 2019;92(July):20190663.

22. Jones LI, Dunn JA. Commentary on: Introduction of an abbreviated breast MRI service in the UK as part of the BRAID trial : practicalities, challenges, and future directions. Clin Radiol [Internet]. 2021;76(6):434-5. Available from: https://doi.org/10.1016/j.crad.2021.02.004

23. Fallenberg E. EUSOBI Diploma [Internet]. European Society of Breast Imaging. [cited 2021 Mar 30]. Available from: https://www.eusobi.org/europeandiploma-in-breast-imaging-edbi/

24. Health Education England. NBIA Training Curriculum [Internet]. National Breast Imaging Academy. [cited 2021 Mar 30]. Available from: https://nationalbreastimagingacademy.org/about-radiology/nbia-fellowship/assessment-guidance/training-curriculum/

25. DeMartini W, Strigel R. Breast MR Course ACR [Internet]. American College of Radiologists. 2021 [cited 2021 Apr 6]. Available from: https://www.acr.org/Lifelong-Learning-and-CME/Education-Center/breast-mr-guided-biopsy

26. Ko ES, Morris EA. Abbreviated magnetic resonance imaging for breast cancer screening: Concept, early results, and considerations. Korean J Radiol [Internet]. 2019;20(4):533-41. Available from: https://doi.org/10.3348/kjr.2018.0722

27. Harding S, Geach R, Jones L. The use of 'Think-Out-Loud' methodology in the development of teaching materials for abbreviated breast Magnetic Resonance Imaging scan (FAST MRI) interpretation, and a comparison of the learning experience of two reader cohorts. Eur J Radiol Open [Internet]. 2019;6(May):220-4. Available from: https://doi.org/10.1016/j.ejro.2019.06.002

28. Kim ES, Cho N, Kim S, Kwon BR, Yi A, Ha SM, et al. Comparison of Abbreviated MRI and Full Diagnostic MRI in Distinguishing between Benign and Malignant Lesions Detected by Breast MRI: A Multireader Study. 2021;22(3):297-307. Available from: https://doi.org/10.3348/kjr.2020.0311

29. EA1141 trial organisers. Abbreviated breast MRI training course and certification [Internet]. Society of Breast MRI (Washington, USA). 2018 [cited 2021 Apr 9]. Available from: http://www.societyofbreastmri.org/Training.html 
30. Wilson R, Liston J, Thomas KG, Hopkins C, Sellars S, Thompson W. Quality Assurance Guidelines for Breast Cancer Screening Radiology: Second edition [Internet]. 2011. Available from:

https://assets.publishing.service.gov.uk/government/uploads/system/uploads/attachment_data/file/764452/Quality_assurance_guidelines_for_breast_c

31. NICE Guidelines. Familial breast cancer: classification, care and managing breast cancer and related risks in people with a family history of breast cancer. National Institute for Health and Care Excellence.

32. Looney PT, Young KC, Halling-Brown MD. MedXViewer: Providing a web-enabled workstation environment for collaborative and remote medical imaging viewing, perception studies and reader training. Radiat Prot Dosimetry [Internet]. 2016;169(1-4):32-7. Available from:

https://doi.org/10.1093/rpd/ncv482

33. Spencer J. Learning and teaching in the clinical environment How doctors teach Experiential learning. Br Med J [Internet]. 2003;326(March):591-4. Available from: https://doi.org/10.1136/bmj.326.7389.591

34. Taylor K, Britton P, O'Keeffe S, Wallis MG. Quantification of the UK 5-point breast imaging classification and mapping to BI-RADS to facilitate comparison with international literature. Br J Radiol. 2011;84(1007):1005-10.

35. Public Health England. Technical guidelines for magnetic resonance imaging (MRI) for the surveillance of women at higher risk of developing breast cancer (NHSBSP Publication No 68) [Internet]. Gov.Uk. 2012. Available from: https://www.gov.uk/government/publications/nhs-breast-screening-usingmri-with-higher-risk-women

36. NHSBSP. NHS Breast Screening Programme Guidance on who can undertake arbitration About Public Health England [Internet]. 2016. p. 1-6. Available from: https://assets.publishing.service.gov.uk/government/uploads/system/uploads/attachment_data/file/548405/Arbitration_guidance.pdf

37. Sickles E, D'Orsi C. ACR BI-RADS ${ }^{\circledR}$ Follow-up and Outcome Monitoring. In: ACR BI-RADS ${ }^{\circledR}$ Atlas, Breast Imaging Reporting and Data System [Internet]. 5th ed. Reston, VA: American College of Radiology; 2013. Available from: https://www.acr.org/Clinical-Resources/Reporting-and-Data-Systems/BiRads/Permissions

38. van Zelst J, Vreemann S, Witt H-J, Gubern-Merida A, Dorrius MD, Duvivier KM, et al. Multireader Study on the Diagnostic Accuracy of Ultrafast Breast Magnetic Resonance Imaging for Breast Cancer Screening. Invest Radiol [Internet]. 2018;53(10):579-86. Available from:

https://dx.doi.org/10.1097/RLI.0000000000000494

39. Lee JM, Ichikawa L, Valencia E, Miglioretti DL, Wernli K, Kerlikowske K, et al. Performance Benchmarks for Screening Breast MR Imaging in. Radiology [Internet]. 2017;285(1):44-52. Available from: https://doi.org/10.1148/radiol.2017162033

40. Rawashdeh MA, Lee WB, Bourne RM, Ryan EA, Pietrzyk MW, Reed WM, et al. Markers of Good Performance in Mammography Depend on Number of Annual Readings. Radiology [Internet]. 2013;269(1):61-7. Available from: https://doi.org/10.1148/radiol.13122581

41. Gur D, Cohen CS, Hakim CM, Hardesty LA, Ganott MA, Perrin RL, et al. The "Laboratory Effect": Comparing Radiologists' Performance and Variability during Prospective Clinical and Laboratory Mammography. Radiology [Internet]. 2008;249(1):47-53. Available from: https://doi.org/10.1148/radiol.2491072025

42. NHS Digital. Breast Screening Services [Internet]. NHS Digital. 2020 [cited 2022 Jan 8]. Available from: https://digital.nhs.uk/services/screeningservices/breast-screening-services

43. Chen Y, James JJ, Cornford EJ, Jenkins J. The Relationship between Mammography Readers' Real- Life Performance and Performance in a Test Set based Assessment Scheme in a National Breast Screening Program. Radiol Imaging Cancer [Internet]. 2020;2(5):1-7. Available from: https://doi.org/10.1148/rycan.2020200016

44. Du T, Zhu L, Levine KM, Tasdemir N, Lee A V, Vignali DAA, et al. Invasive lobular and ductal breast carcinoma differ in immune response, protein translation efficiency and metabolism. Sci Rep [Internet]. 2018;8:1-11. Available from: http://dx.doi.org/10.1038/s41598-018-25357-0

45. Blanks R, Wallis M, Alison R, Given-Wilson R. An analysis of screen-detected invasive cancers by grade in the English breast cancer screening programme: are we failing to detect sufficient small grade 3 cancers? Eur Radiol [Internet]. 2021;31:2548-58. Available from: https://doi.org/10.1007/s00330-02007276-9

46. Saadatmand S, Bretveld R, Siesling S, Tilanus-Linthorst MMA. Influence of tumour stage at breast cancer detection on survival in modern times: Population based study in 173797 patients. BMJ [Internet]. 2015;351:1-9. Available from: https://doi.org/10.1136/bmj.h4901

47. Saadatmand S, Obdeijn IM, Rutgers EJ, Oosterwijk JC, Tollenaar RA, Woldringh GH, et al. Survival benefit in women with BRCA1 mutation or familial risk in the MRI screening study (MRISC). Int J Cancer [Internet]. 2015;137(7):1729-38. Available from: https://doi.org/10.1002/ijc.29534

\section{Figures}




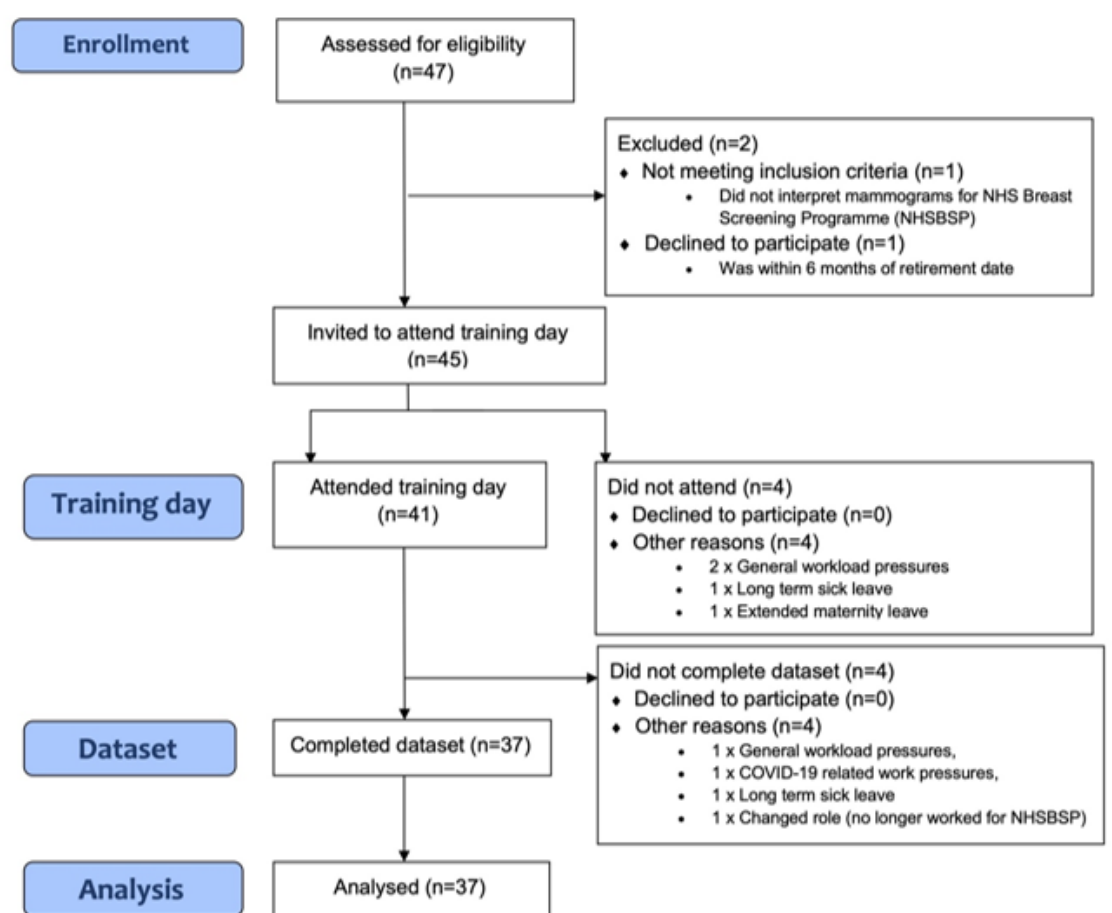

\section{Figure 1}

CONSORT Flow Diagram detailing participation in FAST MRI Reader Training Programme

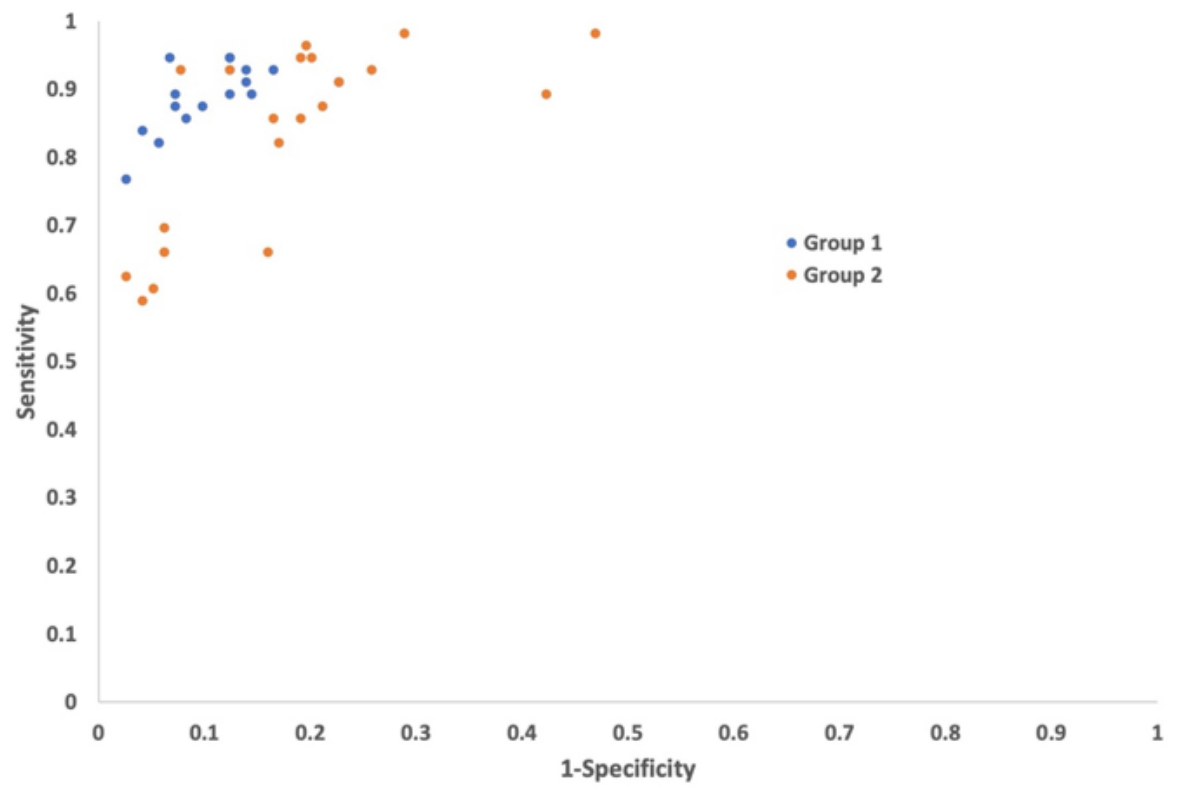

\section{Figure 2}

Point estimates of accuracy in Receiver Operating Characteristic (ROC) space (sensitivity plotted against 1-specificity) for each reader in the study, coded by group (Group 1 = experienced breast MRI readers, Group 2 = mammogram readers who have undergone a single day's training to interpret abbreviated breast MRI) 


\section{Group 1 Group 2}

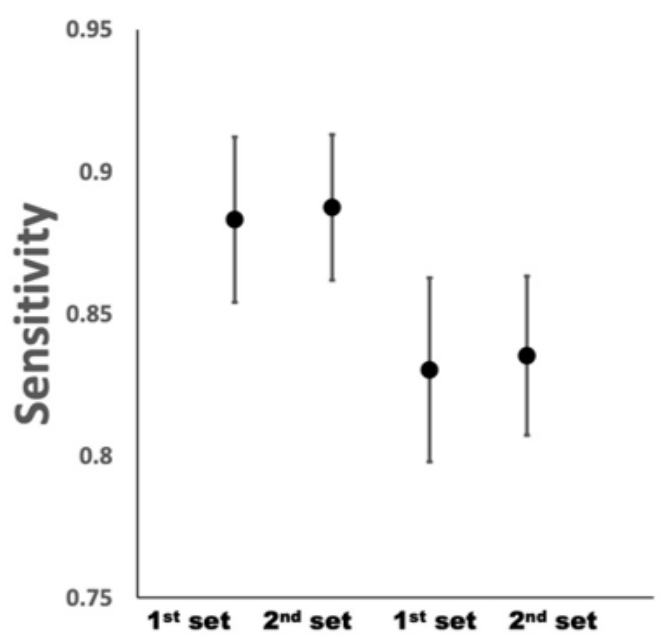

\section{Group 1 Group 2}

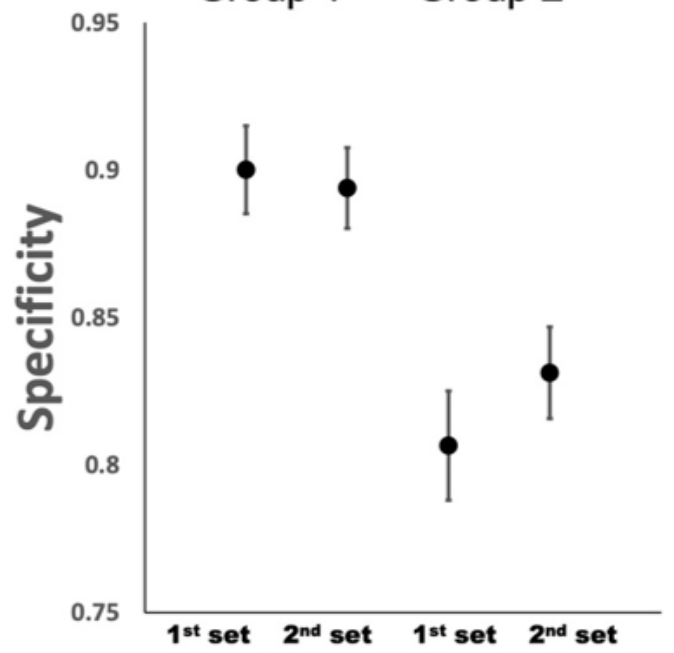

Figure 3

Sensitivity $(95 \% \mathrm{Cl})$ and specificity $(95 \% \mathrm{Cl})$ for the 1 st set of 55 cases compared to 2 nd set of 70 cases, demonstrating improvement in per breast performance (specificity) for Group 2 but not for Group 1

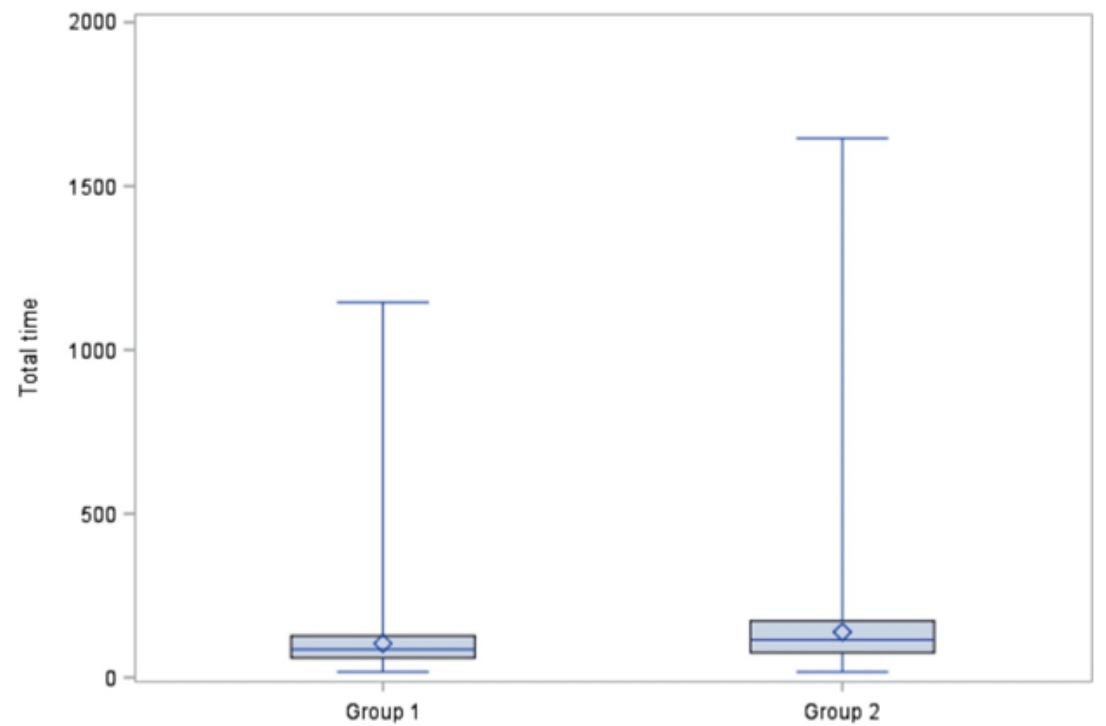

Figure 4

Box and whisker plot for total time taken (seconds) to report each case

The long horizontal blue line represents the median; the top and the bottom of the box represent the 25th and 75th percentiles. The diamond in the box represents the mean. The vertical lines (whiskers) extend to the group minimum and maximum values. The outlier within Group 2 of 10003 seconds has been excluded for this plot. 


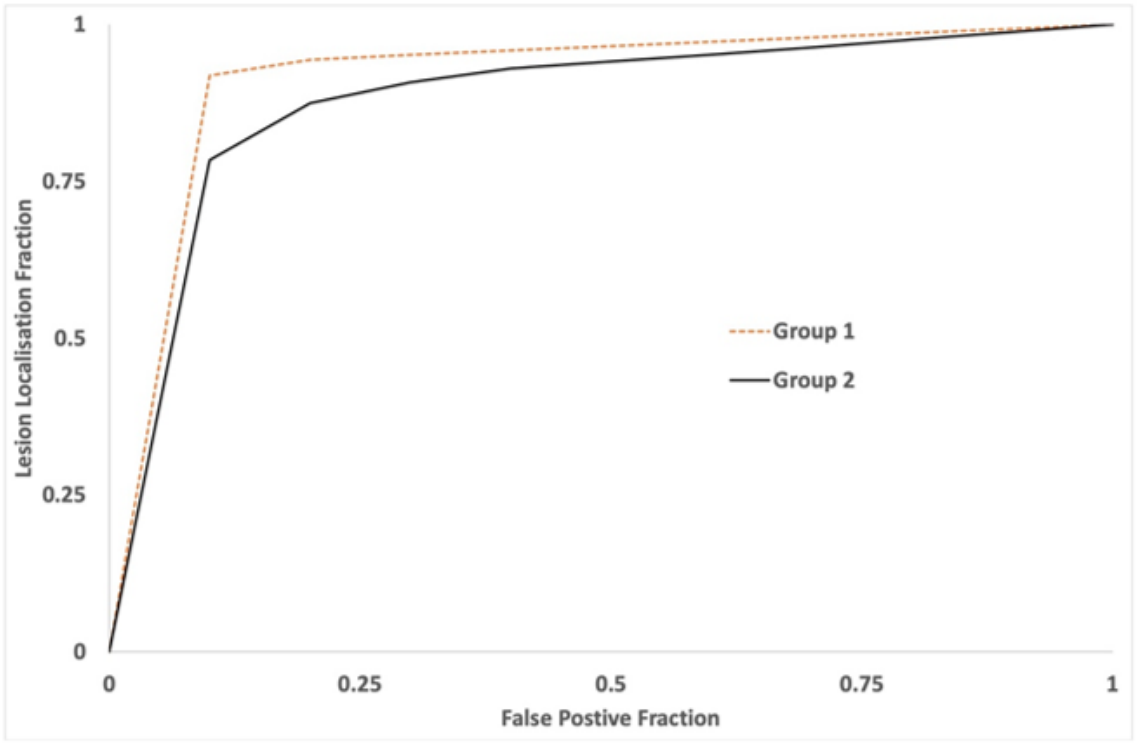

\section{Figure 5}

Per lesion analysis demonstrated graphically as a jackknife alternative free-response receiver operator characteristics (JAFROC) curve

There were 58 biopsy-confirmed cancer lesions in the dataset, equating to a total of 2146 decisions made by the 37 readers. The LLF was overall was $83 \%$ (1783/2146); 86\% (847/986) for the Group 1 readers and 81\% (936/1160) for the Group 2 readers. The reader averaged weighted JAFROC FOM was 0.93 (95\% $\mathrm{Cl} 0.92-0.94)$ overall. The reader averaged weighted JAFROC FOM for Group 1 readers of 0.95 (95\% Cl 0.95-0.96) was significantly higher than for Group 2 (0.91; 95\% Cl 0.89-0.93); $p=0.004)$
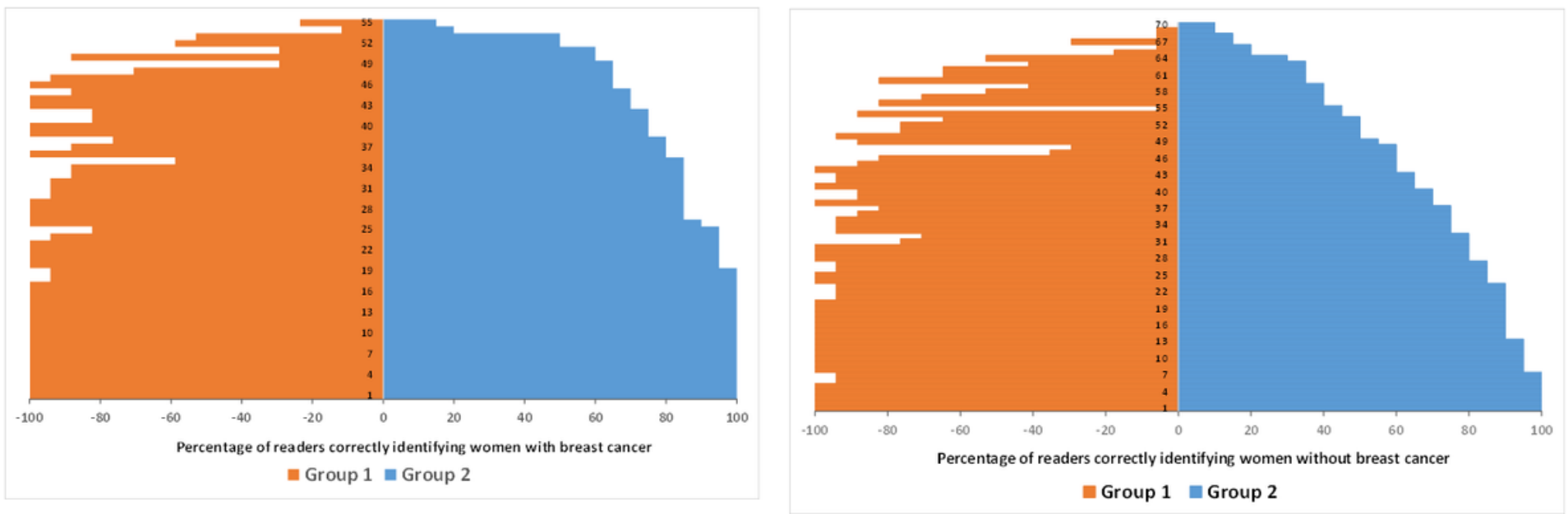

\section{Figure 6}

Per woman/abbreviated breast MRI (abMRI) analysis

a: Illustration of the percentage of readers correctly identifying each of the 55 women with breast cancer

b: Illustration of the percentage of readers correctly identifying each of the 70 women without breast cancer

\section{Figure 7}

\section{a, b \& c: Example cancer case from the dataset}

This $25 \mathrm{~mm}$ diameter Grade 2 carcinoma of no special type (oestrogen receptor positive, progestogen receptor equivocal, Her2 receptor negative and Ki67: $20 \%$ ) was occult mammographically (a) because it was obscured by mammographically dense fibroglandular tissue. It was also difficult to see on the maximum intensity projection (MIP) image of the FAST MRI (b) as it was obscured by background parenchymal enhancement (BPE). However, it is clearly 
seen as a rim enhancing mass on the FAST MRI stack of slices (c), indicated by a yellow arrow. It was correctly identified as a cancer by $17 / 17$ Group 1 readers and 17/20 Group 2 readers during this study.

\section{Supplementary Files}

This is a list of supplementary files associated with this preprint. Click to download.

- ElectronicSupplementaryMaterial.docx 